\title{
Aberrant Ulnar Artery and Ulnar Artery Thrombosis with Nerve Entrapment: A Case Report and Review of Literature
} Nickul N Shah MS-IV, David Roman RPAC and Roland Purcell MD

Interfaith Medical Centre, 1545 Atlantic Avenue, Brooklyn NY 11213, USA

\begin{abstract}
Ulnar artery aneurysms are quite rare discoveries, related to repetitive trauma, vasculitis, anatomic abnormalities, or infections. Typically caused by blunt injury to the ulnar artery and superficial palmar arch, arterial wall damage leads to the formation of an aneurysm. The sensory branch of the ulnar nerve becomes compressed, causing paraesthesia and weakness along the $4^{\text {th }}$ and $5^{\text {th }}$ digits, and resection is required. It is interesting to note, in this particular case, that a high origin and superficial ulnar artery coursed along the forearm and entered along the ulnar side of the hand. A diagnostic and therapeutic approach is necessary to relieve ulnar nerve entrapment. In addition, it is important to establish an early diagnosis prior to any surgical procedures in order to isolate and establish continuous flow to the compromised region. Further studies are required, however, to establish an evidenced-based approach to such anatomic variations with surgical repair.
\end{abstract}

Keywords: Ulnar artery thrombosis; Ulnar nerve compression; Aberrant ulnar artery; Paresthesia

\section{Introduction}

Aneurysmal formations, from excessive dilatation, occurs secondary to damage of blood vessel walls. Aneurysms of the ulnar artery and palmar arch, related to repetitive trauma of the upper extremity, arise from rare circumstances, which affect the hypothenar region of the hand. Adults can experience hand and finger pain from vascular insufficiency and compression of the ulnar nerve. Most traumatic aneurysms are quite common among young males, and sometimes related to congenital abnormalities or infections. In this particular case, a 73-year-old woman underwent an aneurysmal repair after minor trauma and suffered numbness and tingling along the $4^{\text {th }}$ and $5^{\text {th }}$ digits of the right hand. Duplex ultrasound and AV Fistulogram identified an aneurysm a superficial ulnar artery, lying adjacent to the aneurysm. Knowledge of such variations allows for improved care in the fields of radiology, angiography, surgery and the medical sciences.

\section{Case Presentation}

A 73-year-old, non-smoker, Haitian woman was accompanied with her daughter to the community hospital. The patient spoke very little English, but her daughter translated. As per her daughter, the patient complained of numbness, pain and weakness along the right arm, forearm, and wrist. She had visited her home in Haiti two years ago, and believed she unintentionally "bumped her hand on something." The patient has a significant past medical history of hypertension, arthritis of both her hands, bilateral cataracts, and a right sided lumpectomy. Upon examination, a $3-\mathrm{cm}$ pulsatile mass was observed at the ulnar side of the right wrist with a palpable thrill. No obvious signs of trauma were observed. She had numbness and tingling along the $4^{\text {th }}$ and $5^{\text {th }}$ digits of her right hand, but strength was preserved. The Allen's test was conducted, and no significant abnormalities were observed. A Tinel's test was also conducted, but showed hyporeflexia. Pinprick sensation was also diminished along the ulnar distribution of the hand.

Subsequently, she underwent an AV fistulogram (Figures 1-3) with contrast dye, which demonstrated the dilated vessel and aberrant branch of an ulnar artery. There was no disruption of flow proximal or distal to the aneurysm as well as the adjacent artery.

Resection of the right wrist aneurysm was scheduled the same day.
After desensitizing her pain under local anaesthesia, a longitudinal incision was created to expose the aneurysm and its bifurcation points. Intraoperatively, the patient's vitals were stable. During the operation, however, an aberrant ulnar artery (Figure 4) was observed, which was found medial to the aneurysm. Special care was facilitated in order to avoid damage to the artery or the flow to the hand. Multiple microvascular connections were observed with the aneurysm (Figure 5), and again special care was needed to isolate the aneurysm without

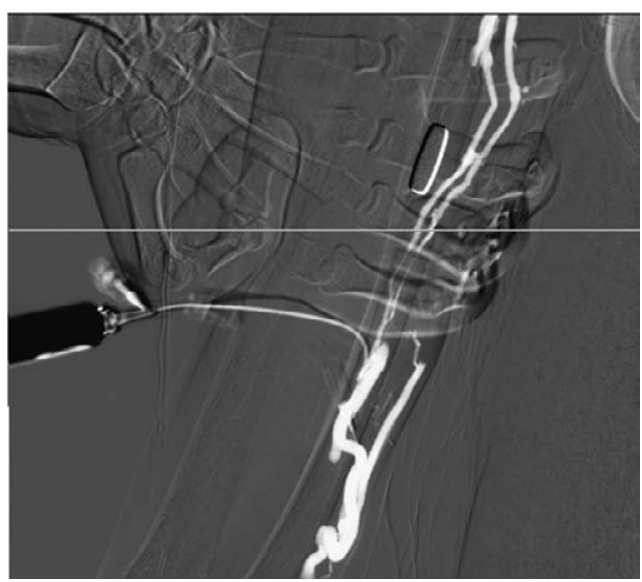

Figure 1: AV fistulogram used to demonstrate the flow.

*Corresponding author: Nickul N. Shah MS-IV, Interfaith Medical Centre 1545 Atlantic Avenue, Brooklyn NY 11213, USA, Tel: +1 718-613-4000; E-mail: Shah.nickul@gmail.com

Received November 03, 2015; Accepted November 14, 2015; Published November 22, 2015

Citation: Nickul N Shah MS-IV, David Roman RPAC, Roland Purcell MD (2015) Aberrant Ulnar Artery and UInar Artery Thrombosis with Nerve Entrapment: A Case Report and Review of Literature. J Vasc Med Surg 3: 230. doi:10.4172/23296925.1000230

Copyright: (c) 2015 Nickul N Shah MS-IV, et al. This is an open-access article distributed under the terms of the Creative Commons Attribution License, which permits unrestricted use, distribution, and reproduction in any medium, provided the original author and source are credited. 
Citation: Nickul N Shah MS-IV, David Roman RPAC, Roland Purcell MD (2015) Aberrant Ulnar Artery and Ulnar Artery Thrombosis with Nerve Entrapment: A Case Report and Review of Literature. J Vasc Med Surg 3: 230. doi:10.4172/2329-6925.1000230

Page 2 of 5

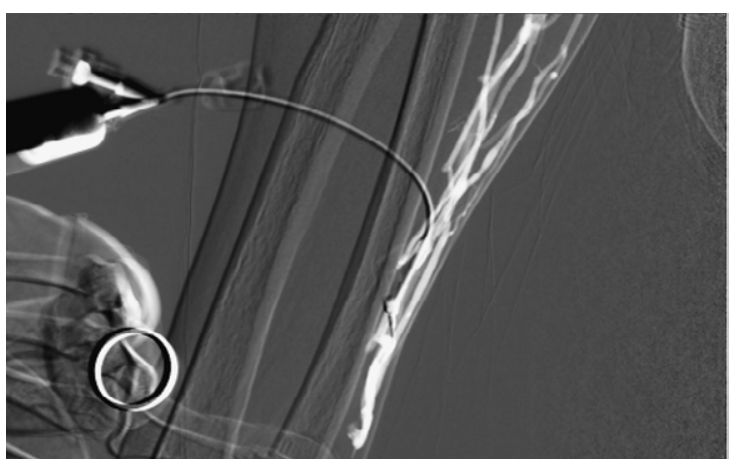

Figure 2: AV fistulogram to demonstrate flow.

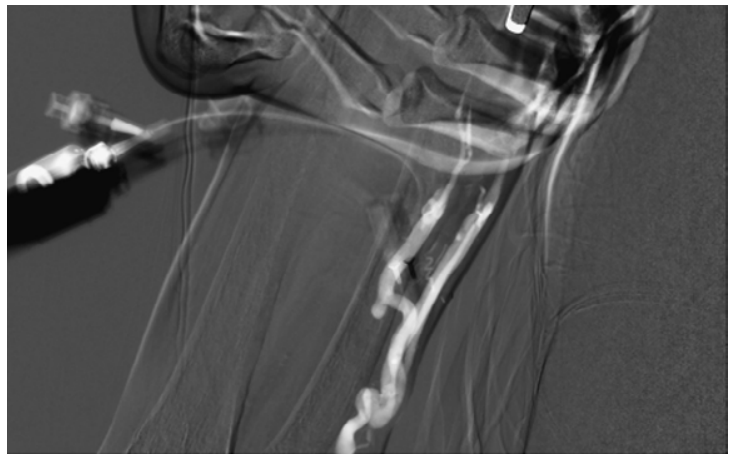

Figure 3: AV fistulogram to demonstrate flow.

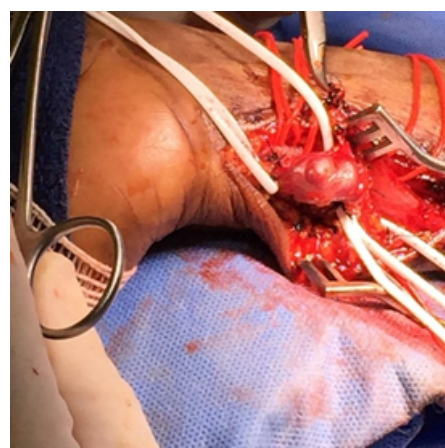

Figure 4: Ulnar artery aneurysm identified and isolated.

excessive blood loss. Ring handle bulldogs were placed in a tangential fashion to occlude flow of the aneurysm and its branch points. The microvascular connections were ligated throughout the procedure while still exposing the aneurysm and the artery of interest. Once the aneurysm was isolated both proximally and distally using additional clamps, it was later resected for histopathological evaluation (Figure 6). The isolated aberrant artery was patent throughout the procedure with continuous flow, as detected by the Doppler Ultrasound. A 3-0 Nylon suture was used to close the wound. The Allen's test was again applied to test for adequacy of blood flow to the hand, and no abnormalities were found. Pinprick sensation and Tinel's test had significantly improved. The patient's vitals remained stable throughout the procedure. No postoperative complications were observed. Pain was adequately controlled in the recovery room. Patient was discharged home the same day, and was advised to follow up one week later.

\section{Discussion}

Aneurysms of the ulnar artery and palmar arch are often found in young males. They arise from repetitive violent or micro- trauma, vasculitis, vascular anomalies or infections of the upper extremity. Patients may present with a spectrum of symptoms including Raynaud's phenomena, digital ischemia, coldness, cyanosis, pallor, pain, gangrene, tenderness of hypothenar area due to compression of ulnar nerve [1]. The severity of symptoms is based on location of the obstruction and the nature of collateral hand circulation. Patients with anomalies to the ulnar artery can cause digital ischemia, thrombosis of the aneurysm, distal embolization to the digital artery. The fourth and fifth digital arteries are most affected.

Traumas to arteries damage the intimal surface and later the thrombogenicty of the vascular endothelium causing arterial thrombosis and ischemia. Aneurysmal formation follows damage to the media. Pathogeensis of dissection is similar to aorta dissection in which shearing of vasovasorum initiates traumatic dissection [2]. Spontaneous formation occurs with acute hypertension and sudden change to blood flow causing intimal tears [2]. Patients complain of pain in the traumatized hand or fingers after a period of days to weeks before ischemic symptoms appear [3].

However, distal ulnar artery and palmar arch aneurysms are uncommon clinical findings associated with Hypothenar Hammer Syndrome, combined with traumatic events [4,5]. Hypothenar Hammer Syndrome is a rare condition, with an incidence up to $14 \%$ and considered a less common etiology of ischemia in the upper extremity from a repetitive or single trauma to the region of the

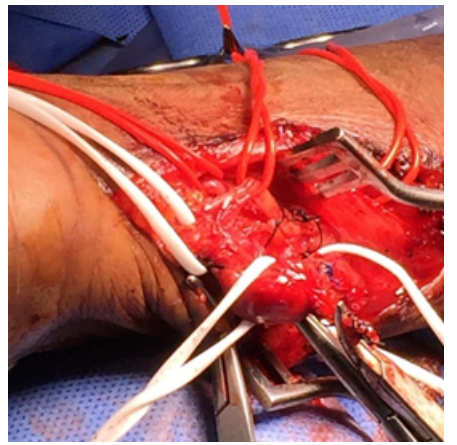

Figure 5: High origin and superficial course of ulnar artery.

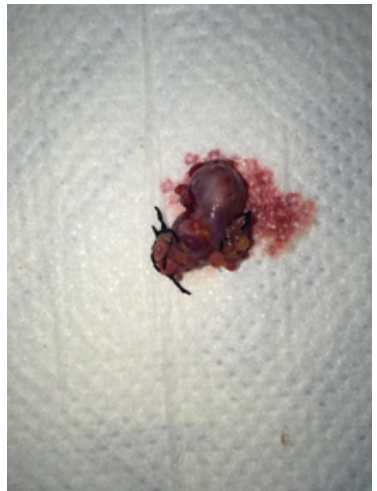

Figure 6: Resected aneurysm sent for histopathologic review. 
hypothenar eminence [6]. Pathogenesis, described by Ablett and Hackett [7], relates to the anatomy of the ulnar artery through the palm and exits the Guyon's canal to form the superficial palmar branch. This arterial segment is susceptible to injury because of limited protection from overlying tissues of the hypothenar muscles. According to an article by Blum et al. [8], both radial artery and ulnar artery provide most of the blood supply to the hand, and additional circulation arise from the median artery or interosseous arterial system. The deep palmar arch and superficial palmar arch are most significant circuits to supply blood to each finger [7,8]. The superficial palmar arch supplies all the fingers and ulnar side of the thumb. The deep palmar arch has a connection with the deep palmar branch of the ulnar artery above the level of the hamulus. Repetitive blunt trauma damages the intima of the ulnar artery as it passes adjacent to the hook of hamate. Subsequently, the artery becomes aneurysmal or thrombose, affecting digital arteries $[9,10]$. Hypothenar hammer syndrome arises because of the anatomic relationship of the sensory branch of the ulnar nerve and superficial branch of the ulnar artery in association with the hamulus [8]. Ferris and his colleagues have suggested a fibromuscular dysplasia etiology, in which the intimal and medial hyperplasia results in a disruption in the internal elastic lamina with areas of stenosis and dilatation [6].

Spontaneous aneurysms of the ulnar artery, a complication of rheumatic vasculitis with systemic symptoms, have been reported since 1958[1]. Seishiro et al. [11] reported a case of a 54-year-old man with a non-traumatic psuedoaneurysm of the proximal ulnar artery with eosinophilia. Kisacik et al. [12] reported a case of a 21-year-old man with ulnar artery aneurysm and history of Behcet's disease. These additional rheumatic vasculitidies have multi-systemic involvement and specific clinical findings. Histological examinations would show lymphocytic infiltration and aneurysmal changes to vessel wall with irregular necrosis and diffuse interstitial fibrosis. Nguyen et al. [13] reported a case of ulnar artery aneurysm (Figure 7) in a patient with Marfan syndrome, a multi system connective tissue disorder with muscolskeletal abnormalities, and cardiovascular manifestation with progressive dilation of ascending aorta causing aortic rupture or dissection. Infectious vasculitidies [2] have also been described, with the most frequent being Hepatitis B virus-related polyarteritis nodosa or Hepatitis C-related mixed cryoglobulinemia [14]. Some bacteria, fungi, or parasites $[15,16]$ cause vasculitis by direct invasion of blood vessels or septic embolism.

This patient most likely had a true spontaneous aneurysm after minor trauma, compressing the ulnar nerve, and disrupting the vessel wall architecture. This patient's history did not fit the criteria for any infectious cause, and no systemic disabling symptoms, including joint pain, were reported. The histologic images for only showed intimal degeneration and thrombus formation with loss of smooth muscle and elastic fibers, and arterial wall thickening with collagen infiltration with granulation tissue, without lymphocyte predominance (Figure 8).

Ulnar neuropathy also known as Guyon Canal Syndrome, a differential diagnosis, shares neurological symptoms the patient experienced. As described by Blum [8], ulnar neuropathy affects athletes involved in cycling (handlebar palsy), in which repetitive and continuous pressure onto the wrist compresses the ulnar nerve. It may also affect workers exposed to frequent vibrations, includes those who work in foundries. Lastly, adjacent masses such as lipomas, anomalous muscles, ulnar artery aneurysms, and hamulus fractures entrap the nerve.

Neurologic symptoms, secondary to nerve entrapment, from an ulnar artery aneurysm affects the superficial or sensory branch of the nerve with sensory loss to the hypothenar eminence, including the fourth and fifth digits [17]. There is tenderness to palpation, and patients may complain of paresthesias, weakness, and tingling that radiates to the fourth and fifth digits [7].

Similar symptoms between arteriovenous fistulas and traumatic fistulas are expressed including thrombosis, stenosis, aneurysm formation and infection [18]. Complications in AVF aneurysms include pain, erosion of the overlying skin with bleeding and infection, steal syndrome, venous hypertension, and high output heart failure with AVF flow $>2500 \mathrm{~mL} /$ minute [19]. This is the result of destruction of vessel wall and replacement of inferior collagenous tissue [19]. After repetitive puncture of the same vessel segment, the wall stress and formation of aneurysm increases as scar tissue replaces vessel wall architecture [19].

Comparing both types of aneurysms, it can be concluded that this patient had a true aneurysm, based on the size of dilation which is three times larger than native size (over $2 \mathrm{~cm}$ )[19]. Patients who experience AVF aneurysm have additional risk factors which include hypertension, hypotension, immunologic disease, diabetes mellitus, arteriolosclerosis, early puncture of AVF site, repeated punctures, and mechanical compression [20,21]. True aneurysm; however take years to occur after fistula creation, with a mean time of 47 months [19]. The patient described did not have chronic renal failure, and an AVF was not indicated. Although she does have hypertension, it contributes more towards a traumatic compressive aneurysm compared to an AV fistula aneurysm.

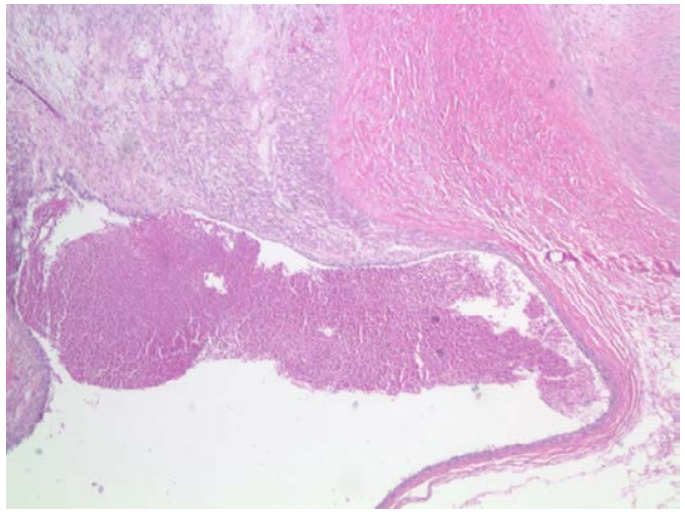

Figure 7: Low magnification of excised aneurysm.

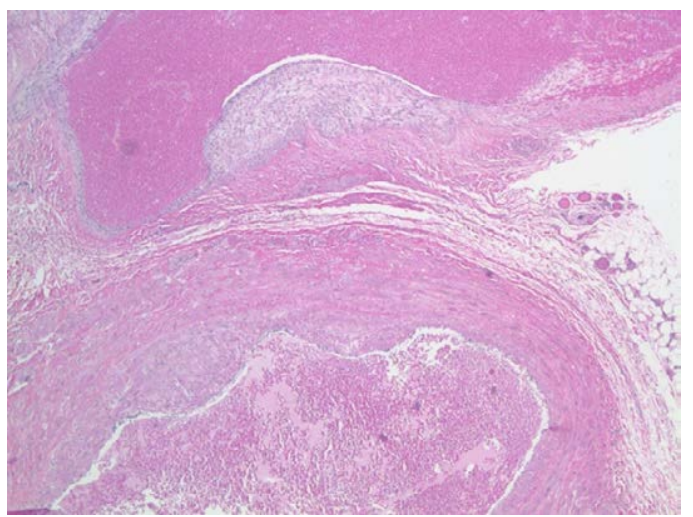

Figure 8: Higher magnification of aneurysm showing collagen infiltration. 
A pulsatile hypothenar mass was present after the formation of the aneurysm without signs of digital ischemia, cyanosis, or atrophic ulcerations. Allen's test assessed the patency of the superficial palmar arch. A positive Allen's test suggests occlusion, stenosis or incomplete development of the superficial palmar arch or distal ulnar artery [7]. In addition, a fistulogram observed a proximal stenosis that may be present [17]. Upon reviewing the literature, a Doppler Ultrasound examination of the extremity and serial arteriograms are useful to evaluate the aneurysm, and differentiate it against other vascular anomalies. For example, fibromuscular dysplasia is ruled out because of absence of a corkscrew sign that demonstrates areas of stenosis and ectasia [22].

According to Ablett and Hackett [7], therapeutic strategies are limited, but consist of smoking cessation, avoidance of further trauma, protective clothing in an industrial occupation, cold avoidance, calcium channel blockers, anticoagulation, and local care of fingers, and pentoxifylline to reduce blood viscosity [19].

Reasons to repair an aneurysm after failed conservative therapy include skin changes such as thinning or erosion, inflammation, pain, and thrombosis with flow impediment [7]. Based on a small number of patients, researchers have agreed that the best outcomes are found in those treated with surgical resection and reconstruction[7] Limitations include retrospective study design, variable mix of thrombosis and aneurysm study design, and treatment of ligation versus venous or arterial grafts $[23,24]$. The advantages include removal of a source embolism, removal of a painful compressive mass over the ulnar nerve [10]. Surgical decompression must be considered to avoid irreversible nerve alterations [25]. The thrombotic form must also be resected to avoid occlusion of digital branches secondary to thromboembolism [26]. Surgical options depend on the presence of adequate perfusion in the hand.

Simple resection is considered with adequate hand perfusion and intact arteries. If there is inadequate flow, ulnar artery reconstruction using microsurgical technique is mandatory using end-to-end anastomosis [10]. Some believe that the ulnar artery should be reconstructed when resected to prevent further complications [10]. In the presented case, the ulnar artery aneurysm (Figure 7) was resected without further reconstruction, as the angiographic evidence of the superficial artery (Figure 5) provided adequate perfusion of the hand, as demonstrated by the Allen test and Doppler ultrasound. If left untreated, local compression, emboli, distal ischemia, or rupture can develop [19,27].

An anatomical variation in the vascular bundle was isolated and evaluated using the Doppler. According to Balla [28], the presence of an ulnar artery of high origin demonstrates a rare anatomical variation. Pulakunta et al. have also reported a variation of a co-existence of a superficial ulnar artery and an adjacent aneurysm of the deep palmar arch in the hand [29]. The arterial course leads to higher risk of damage during vascular surgery. The use of a Doppler demonstrated the patency of both the ulnar artery and superficial branch. Post-surgical evaluation with the Allen's test provided adequate perfusion along the deep palmar arch.

Careful evaluation of its persistent flow prevented its obliteration intraoperatively. Its patency prevented this patient's complications such as ischemia and gangrene. Knowledge of such anatomical variations is very important to all fields of medicine in clinical practice. Physical examination, diagnostic studies, and the evaluation for surgical intervention with restoration of blood flow are necessary to prevent irreversible damage.

\section{References}

1. Makarenko TP, Klimkovich IG (1958) [Spontaneous aneurysm of the ulnar artery as a complication of rheumatic vasculitis]. Khirurgiia (Mosk) 34: 109-111.

2. Dean JC (2007) Marfan syndrome: clinical diagnosis and management. Eur J Hum Genet 15: 724-733.

3. Pagnoux C, Cohen $P$, Guillevin $L$ (2006) Vasculitides secondary to infections Clin Exp Rheumatol 24: S71-81.

4. Mazzaccaro D, Malacrida G, Stegher S, Occhiuto MT, Caldana M, et al. (2012) Ulnar artery aneurysm: case report and review of the literature. G Chir 33: 110-113.

5. Weinand C, Akbari C, O'Donnell S (2011) A high bifurcation of the dorsal branch with dominant superficial palmar branch of the radial artery: a case report of an aberrant radial artery with traumatic aneurysm. J Hand Microsurg 3: 78-81.

6. Nitecki S, Anekstein Y, Karram T, Peer A, Bass A (2008) Hypothenar hammer syndrome: apropos of six cases and review of the literature. Vascular 16: 279282.

7. Ablett CT, Hackett LA (2008) Hypothenar hammer syndrome: case reports and brief review. Clin Med Res 6: 3-8.

8. Blum AG, Zabel JP, Kohlmann R, Batch T, Barbara K, et al. (2006) Pathologic conditions of the hypothenar eminence: evaluation with multidetector $\mathrm{CT}$ and MR imaging. Radiographics 26: 1021-1044.

9. Smith HE, Dirks M, Patterson RB (2004) Hypothenar hammer syndrome: Dista ulnar artery reconstruction with autologous inferior epigastric artery. J Vasc Surg 40: 1238-1242.

10. Monacelli G, Rizzo MI, Spagnoli AM, Monarca C, Scuderi N (2010) Ulnar artery thrombosis and nerve entrapment at Guyon's canal: our diagnostic and therapeutic algorithm. In Vivo 24: 779-782.

11. Sekino S, Takagi H, Kato T, Matsuno Y, Sekido Y, et al. (2005) Nontraumatic pseudoaneurysm of the proximal ulnar artery with eosinophilia. J Vasc Surg 42: $1233-1235$.

12. Kisacik B, Kasifoglu T, Akay S, Yilmaz O, Yilmaz S, et al. (2010) Ulnar artery aneurysm in a patient with Behçet's disease. Rheumatol Int 30: 383-385.

13. Nguyen $D Q$, Murison $M(2006)$ Ulnar artery aneurysm in a patient with Marfan's syndrome. J Plast Reconstr Aesthet Surg 59: 1131-1132.

14. Pagnoux C, Cohen $P$, Guillevin $L$ (2006) Vasculitides secondary to infections Clin Exp Rheumatol 24: S71-81.

15. Inoue T, Otaki M, Wakaki N, Oku H (2001) Mycotic aneurysm of the palmar artery associated with infective endocarditis. Case report and review of the literature. Minerva Cardioangiol 49: 87-90.

16. Bacourt F, Bornet P, Fourcade R, Davous N, Lacombe P (1987) [Aneurysm of the ulnar artery in Streptococcus bovis septicemia. First case]. Presse Med 16: 450 .

17. Lo HY, Tan SG (2007) Arteriovenous fistula aneurysm--plicate, not ligate. Ann Acad Med Singapore 36: 851-853.

18. Konner K, Nonnast-Daniel B, Ritz E (2003) The arteriovenous fistula. J Am Soc Nephrol 14: 1669-1680.

19. Pasklinsky G, Meisner RJ, Labropoulos N, Leon L, Gasparis AP, et al. (2011) Management of true aneurysms of hemodialysis access fistulas. J Vasc Surg 53: 1291-1297.

20. Elseviers MM, Van Waeleghem JP (2003) European Dialysis and Transplant Nurses Association/European Renal Care Association. Identifying vascular access complications among ESRD patients in Europe. A prospective, multicentre study. Nephrol News Issues 17: 61-4.

21. Cavallaro G, Taranto F, Cavallaro E, Quatra F (2000) Vascular complications of native arteriovenous fistulas for hemodialysis: role of microsurgery. Microsurgery 20: 252-254.

22. Cooke RA (2003) Hypothenar hammer syndrome: a discrete syndrome to be distinguished from hand-arm vibration syndrome. Occup Med (Lond) 53: 320324 
Citation: Nickul N Shah MS-IV, David Roman RPAC, Roland Purcell MD (2015) Aberrant Ulnar Artery and Ulnar Artery Thrombosis with Nerve Entrapment: A Case Report and Review of Literature. J Vasc Med Surg 3: 230. doi:10.4172/2329-6925.1000230

23. Dethmers RS, Houpt $P$ (2005) Surgical management of hypothenar and thenar hammer syndromes: a retrospective study of 31 instances in 28 patients. $J$ Hand Surg Br 30: 419-423.

24. De Monaco D, Fritsche E, Rigoni G, Schlunke S, Von Wartburg U (1999) Hypothenar hammer syndrome. Retrospective study of nine cases. J Hand Surg $\mathrm{Br} 24: 731-734$

25. Nemoto $\mathrm{K}$ (1983) [Experimental study on the vulnerability of the peripheral nerve]. Nihon Seikeigeka Gakkai Zasshi 57: 1773-1786.

26. Birrer M, Baumgartner I (2002) Images in clinical medicine. Work-related vascular injuries of the hand--hypothenar hammer syndrome. $\mathrm{N}$ Engl $\mathrm{J}$ Med 347: 339.
27. Karatepe C, Yetim TD (2011) Treatment of Aneurysm of Hemodialysis Access Arteriovenous Fistulas. Turkish Journal of Thoracic and Cardiovascular Surg 19: 566-569.

28. Balla L, Syamala G, Prasa KSN (2015) High Origin and Aberrant Superficial Course of Ulnar Artery: A Case Report. IOSR Journal of Dental and Medical Sciences 14: 7-9.

29. Pulakunta T, Potu BK, Vollala VR, Gorantla VR, Thomas H (2009) Co-existence of superficial ulnar artery and aneurysm of the deep palmar arch in the hand. BratisI Lek Listy 110: 738-739. 